\title{
Pupil size and the pattern evoked visual response
}

\author{
C H HAWKES* AND B STOW $\dagger$ \\ From the Department of Neurology and Electroencephalography, Ipswich Hospital, Ipswich, Suffolk
}

SUMMARY The effect of artificially varying the pupil size upon the pattern evoked visual response was examined in six healthy subjects and six patients with multiple sclerosis who had abnormal visual evoked responses. Slight depression of amplitude was seen whether the pupil was small or large, but the most obvious effect was a reduction of latency with a large pupil and an increase of latency with a small pupil. It is suggested these phenomena relate to the amount of light entering the eye and not any change of acuity.

The flash evoked visual response is known to possess considerable variability which has so far precluded its routine clinical use. Conversely the pattern response is more stable between recording sessions and has substantial clinical value, but may nonetheless be influenced by such factors as field width, square size, luminance and visual acuity. The relevance of pupil size has so far not been examined.

\section{Method}

A black and white chequer-board pattern was displayed to create a $32^{\circ}$ diameter field when viewed at 1 metre. ${ }^{1}$ The individual squares subtended $50^{\prime}$ at this distance and reversed twice per second. The luminance of the white and black squares was 137 and 14 candles/square metre respectively (SEI exposure photometer, Ilford Ltd). Monocular whole field stimulation was performed in each case. The occipital response was detected by four monopolar channels at (1) inion, (2) $5 \mathrm{~cm}$ above the inion, (3) $5 \mathrm{~cm}$ either side of position 2. The reference electrode was at $F_{\mathrm{z}}$ and standard $1 \mathrm{~cm}$ chlorided silver stick-on electrodes were used. The results of 256 reversals per eye were analysed on a Datalab DL 4000 averager and DL 450 microprocessor and simultaneously retained on a Racal Store 7 FM tape recorder. Latency was measured to the first major positive peak (P100) and its amplitude was from here to the preceding negative peak.

*Consultant Neurologist.

†EEG Technician.

Address for reprint requests: $\operatorname{Dr}$ C H Hawkes, Dept. of Neurology, Ipswich Hospital, Heath Road, Ipswich 1P4 5PD.

Accepted 26 August 1980
Pattern evoked responses were obtained from six healthy individuals of either sex (ages 15-30 years) and six subjects with multiple sclerosis (ages 32-45 years), whose $\mathrm{P} 100$ responses were delayed in both eyes. Younger subjects were deliberately chosen for the normal group to avoid any error related to refraction.

The pupil of one eye was constricted with pilocarpine $1 \%$ whilst the other was simultaneously dilated with tropicamide $0.5 \%$ (Mydriacyl) placed into the conjunctival sac. An initial VER was obtained and then repeated after the drugs had acted-typically 30 minutes later. Assessment of visual acuity and pupil size was made before and after the drugs were given and during all measurements particular attention was paid to the consistency of background illumination.

\section{Results}

Healthy patients Pilocarpine constricted the pupil by $1.75 \pm 0.4 \mathrm{~mm}$ (mean $\pm 1 \mathrm{SD}$ ) and diminished acuity typically from $6 / 5$ to $6 / 6$ on the Snellen Chart in all but one case. Changes in latency and amplitude were examined in all four channels but those in channel two were found to be representative and are shown in the graph. The mean change in latency was $4.6 \pm 1.8 \mathrm{~ms}$ and of amplitude $-1.9 \pm 1.7 \mu \mathrm{V}$. Tropicamide dilated the pupil by $4 \cdot 1 \pm 0.9 \mathrm{~mm}$ and caused very slight loss of acuity-never more than three letters of row $6 / 5$. The average change for latency was $-3 \cdot 1 \pm 3: 3 \mathrm{~ms}$ and for amplitude $-1 \cdot 8 \pm 1 \cdot 6 \mu \mathrm{V}$.

Patients Pilocarpine caused a constriction of 1.7 $\pm 0.5 \mathrm{~mm}$ and as associated with no loss of acuity in five and a moderate fall in one $(6 / 9-2$ to $6 / 24+1)$. The average latency change as $+7 \cdot 5$ 


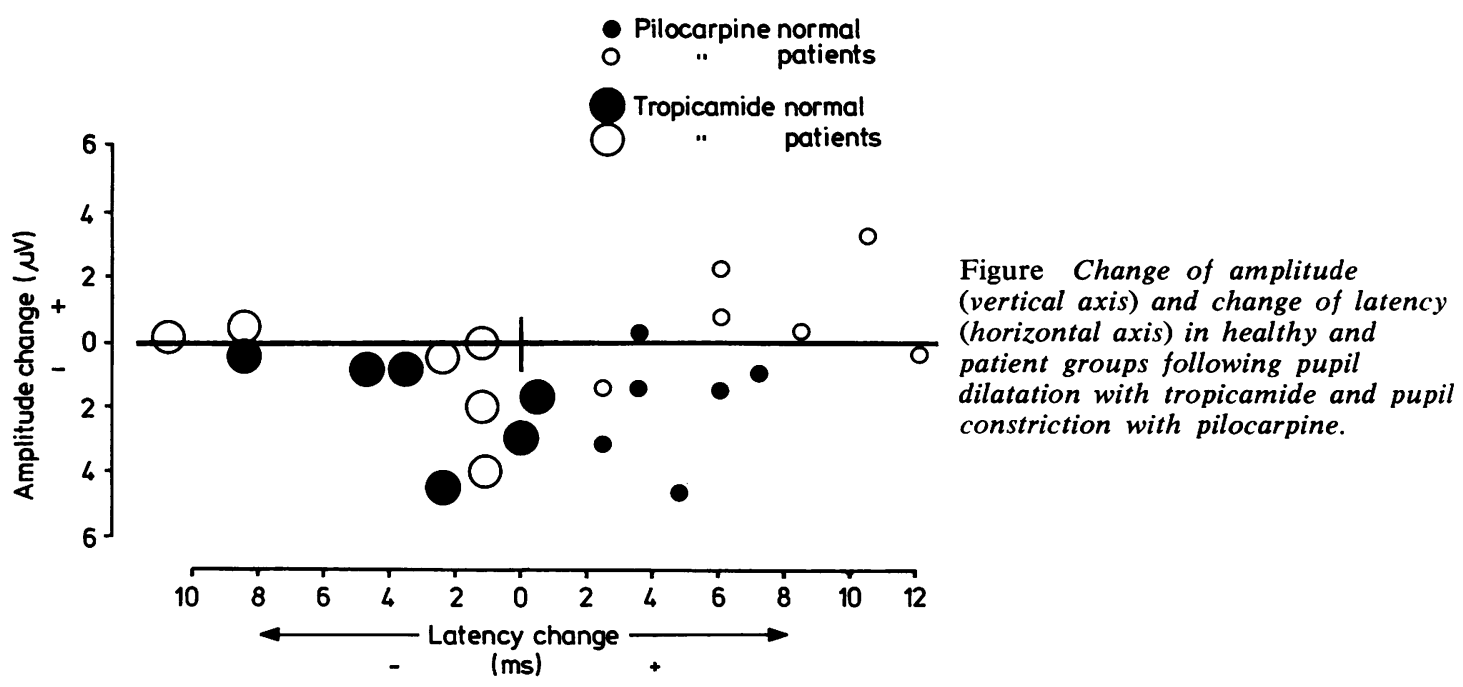

$\pm 3.5 \mathrm{~ms}$ and amplitude altered little $(0 \cdot 9 \pm 1 \cdot 7 \mu \mathrm{V})$. Tropicamide dilated the pupil by $3.8 \pm 0.6 \mathrm{~mm}$, and caused no acuity change in two, a one-row deterioration in three and a two-row fall in one patient. Latency was reduced in all subjects but amplitude again changed little, resulting in a mean shift of $-4 \cdot 2 \pm 4 \cdot 3 \mathrm{~ms}$ and $-1 \cdot 2 \pm 1 \cdot 7 \mu \mathrm{V}$.

\section{Discussion}

In general the changes observed were small but the trend was clearly towards increase of latency with pupil constriction and decrease of latency with pupil dilatation. Both drugs tended to cause slight depression of P100 amplitude. There was no obvious difference in response between the patient and normal groups, except that larger individual shifts were seen in multiple sclerosis patients. Part of the adverse effect on both amplitude and latency may be secondary to deterioration of acuity ${ }^{2}$ although we found no correlation between these factors. Acuity change could not account for the decrease in latency observed with mydriasis. We suspect that the fundamental cause of latency change relates to the quantity of light entering the eye. It has been shown ${ }^{3}$ that a logarithmic relationship exists between stimulus brightness and latency. We did not think any shift in latency was due to degrading of P100 wave shape when its amplitude fell. Although major change was not seen following either drug it is suggested that caution is observed when obvious pupil asymmetry is present as, for example, in patients with unilateral Horner's syndrome or oculomotor palsy.

\section{References}

1 Halliday AM, McDonald WI, Mushin J. Delayed visual evoked response in optic neuritis. Lancet 1972; 1:982-5.

2 Collins DWK, Carrol WM, Black JL, Walsh M. Effect of refractive error on the visual evoked response. Br Med J 1979; 1:231-2.

3 Halliday AM, McDonald WI, Mushin J. Delayed pattern evoked responses in optic neuritis in relation to visual acuity. Trans Ophthalmol Soc UK 1973; 93:315-24. 